2018-06

\title{
Confidentiality, anonymity and amnesty for midwives in distress seeking online support Ethical?
}

\section{Pezaro, S}

http://hdl.handle.net/10026.1/16319

\section{$10.1177 / 0969733016654315$}

Nursing Ethics

SAGE Publications

All content in PEARL is protected by copyright law. Author manuscripts are made available in accordance with publisher policies. Please cite only the published version using the details provided on the item record or document. In the absence of an open licence (e.g. Creative Commons), permissions for further reuse of content should be sought from the publisher or author. 


\title{
Confidentiality, anonymity and amnesty for midwives in distress seeking online support - Ethical?
}

\author{
Sally Pezaro and Wendy Clyne \\ Coventry University, UK \\ Clare Gerada \\ The Hurley Group, UK
}

\begin{abstract}
Background: Midwife health is intrinsically linked to the quality of safe patient care. To ensure safe patient care, there is a need to deliver emotional support to midwives. One option that midwives may turn to may be a confidential online intervention, instead of localised, face-to-face support.

Research design: Following the Realist And MEta-narrative Evidence Syntheses: Evolving Standards publication standards, this realist synthesis approach explores the ethical considerations in permitting confidentiality, anonymity and amnesty in online interventions to support midwives in work-related psychological distress. An iterative search methodology was used to select nine papers for review. To assimilate information, papers were examined for ideas relating to ethical dimensions of online interventions to support midwives in work-related psychological distress. This review takes a narrative approach.

Findings: Online interventions can support the development of insight, help seeking and open discussion. Additionally, Internet support groups can become morally persuasive in nature. Anonymity and confidentiality are both effective and therapeutic features of online interventions when used in collaboration with effective online moderation. Yet, ethical dilemmas remain where users cannot be identified.

Discussion: Confidentiality and anonymity remain key components of successful online interventions. However, sanctioning the corollary component of amnesty may provoke moral discomfort for those seeking immediate accountability. For others, amnesty is seen as essential for open disclosure and help seeking. Ultimately, the needs of midwives must be balanced with the requirement to protect the public and the professional reputation of midwifery.

Conclusion: In supporting midwives online, the principles of anonymity, confidentiality and amnesty may evoke some resistance on ethical grounds. However, without offering identity protection, it may not be possible to create effective online support services for midwives. The authors of this article argue that the principles of confidentiality, anonymity and amnesty should be upheld in the pursuit of the greatest benefit for the greatest number of people.
\end{abstract}

\section{Keywords}

Health workforce, Internet, intervention, midwives, occupational, psychological distress, stress, ethics

Corresponding author: Sally Pezaro, Centre for Technology Enabled Health Research (CTHER), Faculty of Health and Life Sciences, Coventry University, Coventry CVI 5FB, UK.

Emails: sally@pezaro.co.uk; sallypezaro@gmail.com; pezaros@uni.coventry.ac.uk 


\section{Introduction}

Commentators have reported that working within modern healthcare services is difficult for staff, as unhealthy working cultures and traumatogenic environments persist. ${ }^{1-3}$ Healthcare staff may find it difficult to disclose ill health or divulge that they may be struggling to cope in the workplace. ${ }^{4}$ Additionally, some individuals may not recognise, or understand mental ill health in themselves. ${ }^{5,6}$ As the wellbeing of healthcare staff directly correlates with the quality of patient care and safe clinical practice, ${ }^{7}$ this has implications for delivery and quality of maternity care, in addition to the impact upon individual staff members.

\section{Rationale for review}

A national maternity review has highlighted that midwives are more likely to report feeling pressured at work than most other healthcare staff. ${ }^{8}$ In providing support, the ethical considerations in relation to online interventions to support midwives in work-related psychological distress have yet to be explored. Midwives can be reluctant to seek help for fear of stigma, and face-to-face 'debriefing' discussions after significant events can have a punitive feel. ${ }^{9-11}$ There has also historically been a reluctance to report poor performance due to a general tolerance of mistakes, fear of inaction and a fear of adverse repercussions. ${ }^{12}$ Those who prefer to engage in online support rather than traditional real-world services have historically done so because of stigma, shame, linguistic barriers and inconvenience. ${ }^{13,14}$ As such, an online intervention which offers anonymity, confidentiality and their corollary, amnesty, may be the preferred option for midwives in place of face-to-face support. Yet, the ethical considerations associated with the provision of online services to effectively support midwives in distress have yet to be explored.

Medical ethics also point to both anonymity and confidentiality as key factors required in the facilitation of care, because without the promise of confidentiality and anonymity, those in need of help may not be adequately trusting to reveal crucial information. ${ }^{15}$ This would consequently undermine the delivery of appropriate care ${ }^{16-18}$ However, midwives are professionally accountable for patient care, their own health and fitness to practise. As such, the provision of anonymity, confidentiality and their corollary, amnesty, in this particular case, require further ethical exploration and debate.

Society has seen many successful episodes where a period of amnesty has been granted for the benefit of all. Examples of this include gun, drug and knife amnesties, where individuals can admit to an offence without any risk of reprisal. ${ }^{19-21}$ In the context of healthcare, there have also been successful disposal of unwanted medication properly ('DUMP') campaigns, where unwanted medicines have been relinquished to pharmacies for safe disposal without the fear of judgement or retribution. ${ }^{22}$ The benefits of these periods of amnesty are that those in need of help may take a unique window of opportunity to seek help, where they may not otherwise have done so.

Specific strategies may be used within online interventions to support and encourage face-to-face help seeking and open disclosure, such as the Pathways Disclosure Model. ${ }^{23}$ The Pathways Disclosure Model specifically describes how online disclosure can become part of a process for those who are in a precontemplative stage of change to follow a non-threatening pathway towards seeking face-to-face help and disclosure. In this context, the anonymity and confidentiality afforded by an online intervention offers users a unique opportunity to covertly sample the helping process, which leads to a greater willingness to participate in help-seeking activities.

Within the Pathways Disclosure Model, it is the safety of absolute anonymity and confidentiality which remain the key to sustainability in recovery. ${ }^{24}$ Although this model has only previously been applied to those with gambling and alcohol addictions, this model could also be applied to supporting midwives using an online intervention during work-related psychological distress. Figure 1 demonstrates the various steps towards face-to-face help and disclosure as outlined by Pathways Disclosure Model. 


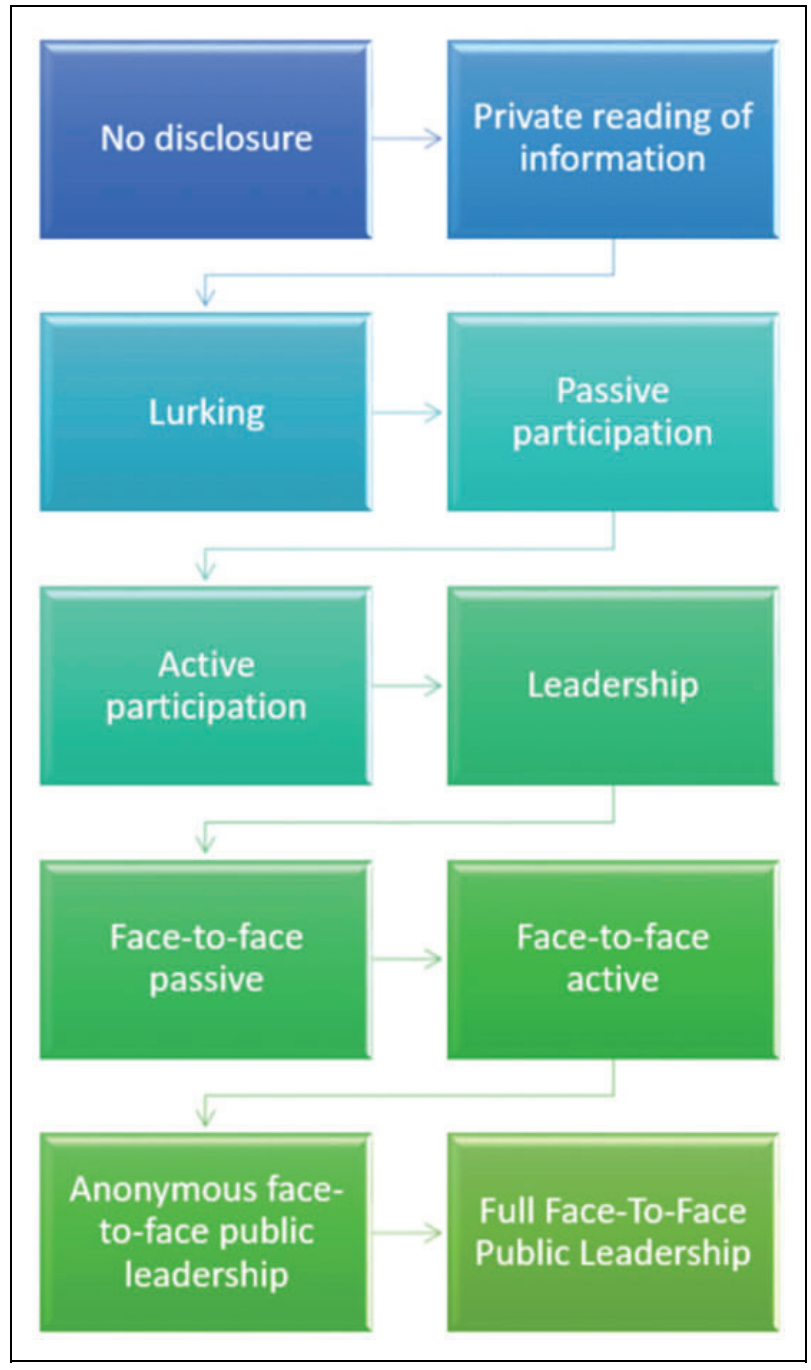

Figure I. The Pathways Disclosure Model in computer-mediated communication.

\section{Objectives and focus of review}

Working while feeling too unwell to perform clinical duties adequately is incompatible with safe and effective clinical practice. ${ }^{25}$ Yet, $68 \%$ of the United Kingdom's National Health Service ${ }^{26}$ staff have reported doing so. Globally, midwives report concerning levels of work-related psychological distress. ${ }^{27}$ Online support for midwives experiencing work-related psychological distress is one potential support provision for this group, providing 24-h access and a wide reach.

This realist synthesis review outlines and explores three ethical considerations in the development of online interventions to support midwives in work-related psychological distress to inform ethical decisionmaking. ${ }^{28}$ These are namely confidentiality, anonymity and amnesty. This article relates these ethical considerations specifically to midwives, as midwives are a professional group unique in the fact that they 
work within an understaffed area of high litigation, where their clinical workload is becoming increasingly complex. We explore these ethical considerations in order to facilitate moral decision-making and generate further dialogue.

The overriding question for this review was, "What are the ethical considerations associated with the provision of confidentiality, anonymity and amnesty in online interventions to support midwives in workrelated psychological distress?'

\section{Background}

It is widely recognised that midwives can experience psychological distress while caring for women and their families. ${ }^{27,29-32}$ The paucity of attention given to the wellbeing of the healthcare professional has been identified as the missing response in staff management across the globe. ${ }^{33-36}$ Specifically, midwives may be at an increased risk of psychological distress due to the unique and traumatic work environments they experience. $^{35,37}$

Ethically, midwives are entitled to a healthy and psychologically safe professional workplace. ${ }^{27,38}$ Yet, midwifery is sometimes based upon a culture of service and sacrifice, which may have historically been prioritised above the individual rights of midwives, and midwives in need of support are often met with inadequate provision. ${ }^{39-41}$ Online interventions that prioritise the needs of midwives in psychological distress may be one option midwives may turn to for support, in line with other populations. ${ }^{42} \mathrm{Such}$ interventions may have the potential to become a powerful tool in improving midwife health and wellbeing. This may in turn protect the public more widely and improve patient care and the quality of safer healthcare services for all. ${ }^{7}$

There is a strong and recent evidence to support the implementation of online psychotherapeutic interventions, which have proved beneficial in providing effective support for other populations in psychological distress. ${ }^{43-45}$ One such emerging online intervention, which one of the authors has an association with - Big White Wall, offers confidential support to thousands of individuals with mental health problems in the online space. An effective and therapeutic online intervention can be defined as one where members are able to communicate, find information, engage and navigate the software with ease. $^{46}$

Some of the benefits of providing support online rather than within a face-to-face scenario are increased accessibility, identity protection and comfort for users. ${ }^{47}$ In an online environment, the benefits of anonymity for vulnerable online users include a significant disinhibition effect, increased feelings of safety and an increased ability for the user to speak openly and honestly for the purpose of developing a therapeutic connection. ${ }^{47}$ For midwives, this could mean speaking openly for the purpose of recovery and help seeking, which could in turn improve the safety and quality of maternity services.

\section{Confidentiality}

Confidentiality is a mutual understanding between two or more parties, where it is the belief of the sender that his or her information will not be shared, and the promise of the receiver to protect and not disseminate the information shared. ${ }^{48}$ For midwives, confidentiality is a professional obligation and can only be broken in the interests of patient and public safety. Confidentiality in the context of an online intervention would mean that users would be expected to keep the identities of individual names, organisations and places confidential. In this context, providing confidentiality to midwives online will also inhibit other users from reporting concerns to professional regulators, as all users remain unidentifiable.

Confidentiality and anonymity in combination are particularly important to help those needing support with suicidal ideation. ${ }^{49}$ However, confidentiality may be legitimately broken if a person is at risk of 
harming themselves. As this would conflict with the provision of anonymity, there is an ethical decision to be made with regard to how this trade-off might be managed. It has been proposed that for those feeling vulnerable, allowing for anonymous and confidential contact and support online may be the optimal method of engagement. ${ }^{50}$ This may be because those in distress often avoid professional help, and online services can provide anonymity, confidentiality, a sense of immediacy and are highly acceptable to younger people. ${ }^{51-53}$ Additionally, research has shown that those at higher risk of suicidal ideation may be more likely to engage with online support. ${ }^{54}$ Therefore, midwives in severe distress may prioritise a confidential online environment to access support, in favour of help avoidance.

Providers of online support interventions may not have the ability to assess the mental state of the participant or intervene in a time of crisis. This is of concern as some virtual environments can be emotionally dangerous for the user. ${ }^{55}$ Any mitigation of risk and harm must be balanced with the benefits associated with supporting midwives to enjoy psychologically safe professional journeys in pursuit of safer maternity services.

\section{Anonymity}

Anonymity has three distinct features: identity protection, action anonymity and visual anonymity. ${ }^{56}$ Identity protection allows a real-world entity to remain unidentified, action anonymity enables a realworld entity to feel 'unknown' by their actions, and visual anonymity relates to a real-world entity having his or her appearance go unnoticed. ${ }^{57}$ Without anonymity, many online activities could become potentially risky to users, as users may become reluctant to share their thoughts openly for fear of stigma, punitive action and/or identification. ${ }^{57-59}$ Encouraging the disclosure of shameful symptoms and related behaviours could be associated with positive outcomes. ${ }^{60,61}$ Therefore, the principle of anonymity could be considered for online interventions designed to support midwives and encourage them to speak openly.

Anonymity in the context of online interventions to support midwives in work-related psychological distress would mean that midwives would be able to experience full identity protection as they interact. This anonymity would be given with the intention of promoting positive therapeutic engagement and help-seeking behaviours. This is significant, as the key to achieving a positive disclosure and a request for real-world help may correlate with the relative amount of anonymity participants are afforded. $^{14,23}$

Nevertheless, in an anonymous cyber space, obligation and accountability can be challenging to achieve where individual users cannot be identified. As the purpose of an online intervention is to support its users, it may be that an online intervention designed to support midwives would not seek to enforce or achieve accountability in this context, particularly given that other channels and processes exist to achieve accountability and uphold professional conduct. We refer to the concept of accountability as

taking responsibility for one's judgments, actions, and omissions as they relate to life-long learning, maintaining competency, and upholding both quality patient care outcomes and standards of the profession while being answerable to those who are influenced by one's nursing practice. ${ }^{62}$

\section{Amnesty}

Amnesty arises as the corollary component of both confidentiality and anonymity. Amnesty is a period of forgiveness, where a crime or misdeed is forgiven, forgotten or 'pardoned' ${ }^{63,64}$ Amnesty in the context of an online support intervention would mean that midwives would be able to disclose an impairment or work-related issue of concern, without fear of retribution or regulatory referral for 
the purpose of help seeking and disclosure. However, midwives have a professional duty to disclose any unsafe practice to their regulator. Should a midwife disclose something of concern online but fail to inform the regulator, this could put patients at risk of further harm and damage the reputation of the profession. As such, some might argue that an amnesty should not be used for midwives in any context.

For doctors in psychological distress, punitive blame cultures and policies often prevent the disclosure of episodes of ill health, addiction and psychological distress. ${ }^{65-68}$ At times, a doctor's insight into the need for help and treatment can also be diminished. ${ }^{68}$ As midwives report similar levels of psychological distress and punitive blame cultures within the workplace, this set of circumstances may be equally apparent in midwifery populations. This may in turn result in a reluctance to seek help or speak openly, which would paradoxically put patients at risk if a compromised healthcare professional continues to practise while they are unfit to do so. ${ }^{69}$ As such, a therapeutic space which permits amnesty may encourage help-seeking behaviours, positive disclosures, a sense of catharsis, real-world behaviour change, reflection and emotional disclosure for midwives in distress. ${ }^{70}$ This journey may also be mapped against the pathways to disclosure model. ${ }^{23}$

Amnesty agreements may provoke moral discomfort. The Council for Healthcare Regulatory Excellence requires the Nursing and Midwifery Council to be seen to protect the public as a primary aim before supporting the wellbeing of the workforce. ${ }^{71} \mathrm{We}$ also recognise that amnesty agreements for healthcare professionals may not be favoured by patients and the public.

\section{Methods}

\section{Aims}

A realist synthesis review involves refining theories and thoughts as the evidence emerges from the literature. $^{72}$ The aim of this realist synthesis review is to explore three ethical considerations in relation to the development of online interventions to support midwives in work-related psychological distress. These ethical considerations are, namely, the provision of confidentiality, anonymity and amnesty.

\section{Rationale for using realist synthesis methodology}

Realist synthesis reviews have an exploratory rather than an evaluative focus. This realist review explores ethical considerations in relation to the development of an online intervention to support midwives in work-related psychological distress, which may require the principles of confidentiality, anonymity and amnesty. Initial scoping searches did not reveal any literature in relation to these principles in this context. This illustrated to us that minimal consideration has been given to this topic previously. As such, we recognised that there was a need to incorporate a range of literature within this review, including grey literature.

Other review methods, such as systematic reviews rely on trial data and effect sizes. These factors are not appropriate to the question currently under study. Therefore, we aimed to look for discursive accounts of the issues involved. A realist synthesis review is appropriate for this task, because its methodology provides an opportunity to identify a number of avenues that might be explored, discussed and explained. ${ }^{73}$ This methodology also has the ability to provide a rationale for synthesising complex ideas swiftly, and to build explanations as to what may work for whom, under what circumstances and why. ${ }^{72}$ This review may also be useful for others, as a realist review is suggested to be more likely to contribute to policy makers' and practitioners' 'sense-making'. ${ }^{72,74}$ 


\section{Search strategy}

This literature search took place between 2 November and 23 December 2015. An iterative search methodology following the Realist And MEta-narrative Evidence Syntheses: Evolving Standards (RAMESES) publication standards for realist syntheses was used. ${ }^{73}$ We began by conducting a background review of the literature, and then a progressive search clarified the scope of the review. Subsequently, a search for evidence was conducted, as prescribed by realist review methodology. ${ }^{72}$ First, Academic Search Complete, Cumulative Index of Nursing and Allied Health Literature (CINAHL) with Full Text, MEDLINE and PsycINFO were searched concurrently for key papers of relevance. Subject headings were used where possible, as were related free text terms and proximity operators.

Search terms were chosen following a brief and scoping review of the literature in relation to midwives in work-related psychological distress, ethical considerations and online interventions. The main search terms were broad and were combined with the AND Boolean operator or combined with the OR Boolean operator as follows:

internet support groups 'AND' ethical issues 'AND' ethics, online interventions 'OR' therapy 'AND' anonymity 'AND' ethics, online communities 'OR' social networks 'AND' peer support interventions 'AND' conduct, virtual communities 'AND' anonymity on the internet 'AND' confidentiality, online intervention 'AND' stigma 'AND' help seeking behaviour, midwives 'OR' midwife 'OR' midwifery 'AND' amnesty. Primary search terms were restricted to the abstract search field, and secondary search terms remained open in scope.

All papers published in English between 1999 and 2015, and all article types were considered for inclusion. In all, 66 papers were retrieved overall, 6 exact duplicates were then removed, leaving 60 papers in total for review. Abstracts, titles and full texts were then scrutinised for their suitability for inclusion and relevance to the review's key themes of confidentiality, anonymity and amnesty.

\section{Inclusion and exclusion criteria}

Studies must shed light upon any ethical aspects which relate to either confidentiality, anonymity or amnesty within online interventions. This includes studies which relate to broadly comparable vulnerable populations. Studies must have been published between 1999 and 2015 in order to reflect a contemporary view of online ethics and midwifery practice. All types of literature and studies will be considered for inclusion due to an anticipated low yield of relevant papers.

\section{Selection and appraisal of documents}

The 60 papers retrieved through this search strategy were initially examined by the primary researcher. Paper titles and abstracts were screened for any relevance to the key themes selected for this review. Articles that clearly did not meet the inclusion criteria were excluded, and any ambiguous papers were read more comprehensively through an iterative process of review. The remaining papers of relevance were then read in their entirety as the inclusion criteria's were re-applied. Final paper selections were then made and agreed with the research team.

The relevance of each paper was judged by its ability to elucidate upon any aspect of either confidentiality, anonymity or amnesty in relation to online interventions designed to support healthcare professionals in distress. The rigour of each paper was judged from a 'fitness for purpose' perspective in line with the realist synthesis approach. ${ }^{73,75}$ Nine papers were chosen for inclusion. Others were omitted either due to their irrelevance to the subject matter, or due to their focus being upon adolescents or elite athletes, rather than comparable groups. 


\section{Data extraction, analysis and synthesis}

The research team assimilated information by annotation rather than 'extracting data', following realist synthesis methodology. ${ }^{75}$ Papers were examined for ideas relating to ethical dimensions of online interventions to support midwives in work-related psychological distress. The synthesis of findings was then related back to the underlying research questions of the review.

This review takes a narrative approach. The findings of the review are presented as a synthesis of evidence. This synthesis explores the ethical considerations in relation to online interventions to support midwives in work-related psychological distress and the key themes of this review - confidentiality, anonymity and amnesty.

\section{Results}

Nine papers were selected following the approach outlined above. Papers included were discursive in nature, ${ }^{47,76-78}$ mixed-method cohort studies, ${ }^{79}$ content analyses, ${ }^{80,81}$ one case study ${ }^{82}$ and theoretical guidance papers. ${ }^{55,83}$ None of the papers retrieved related to midwives or midwifery; therefore, the research team extracted themes of salience in relation to those groups most similar to midwifery populations, including vulnerable groups comparable to midwives in psychological distress. Figure 2 outlines the process for paper selection. A summary of the papers selected for this realist synthesis review can be found in Table 1.

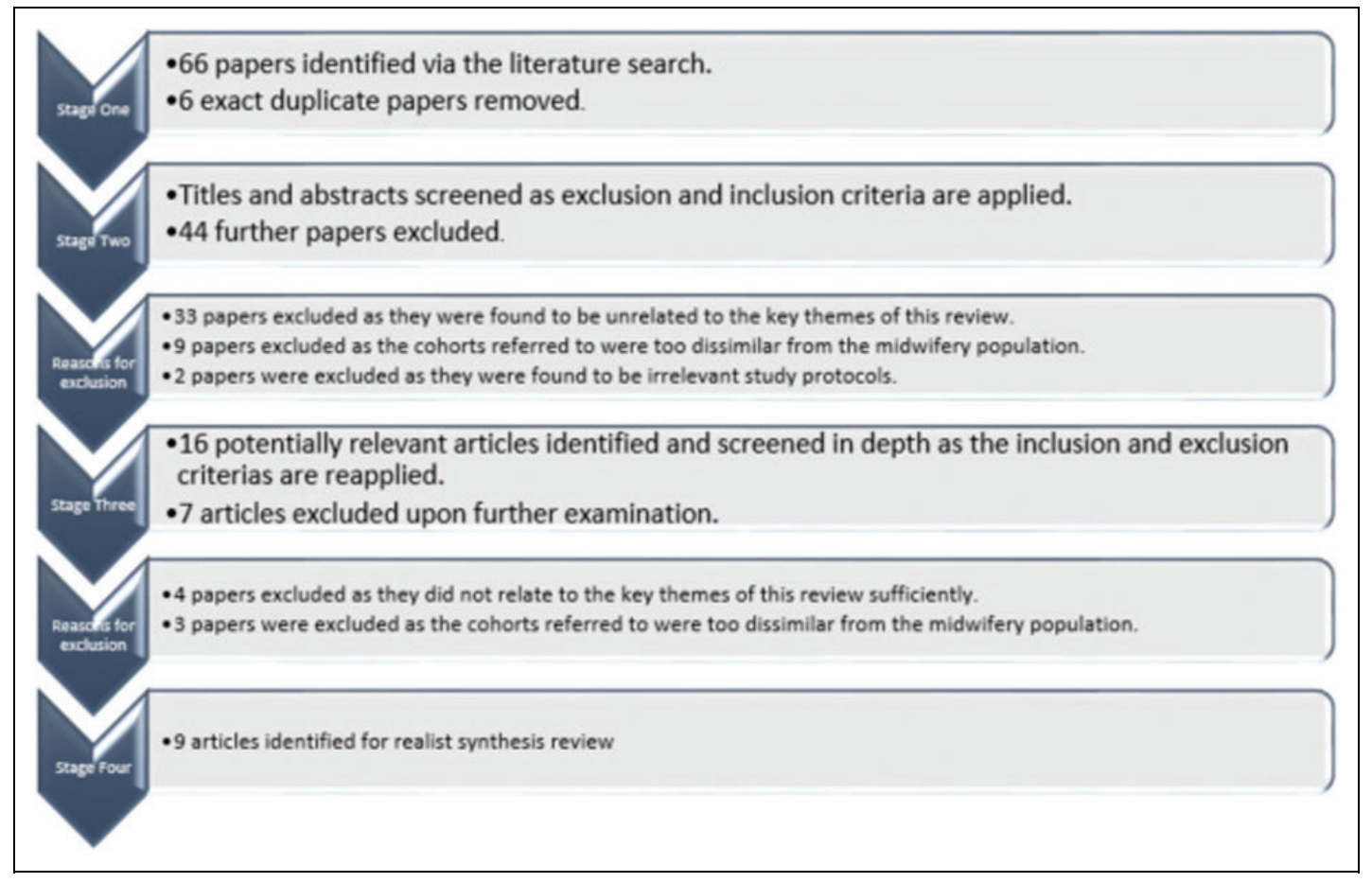

Figure 2. Process of paper selection. 


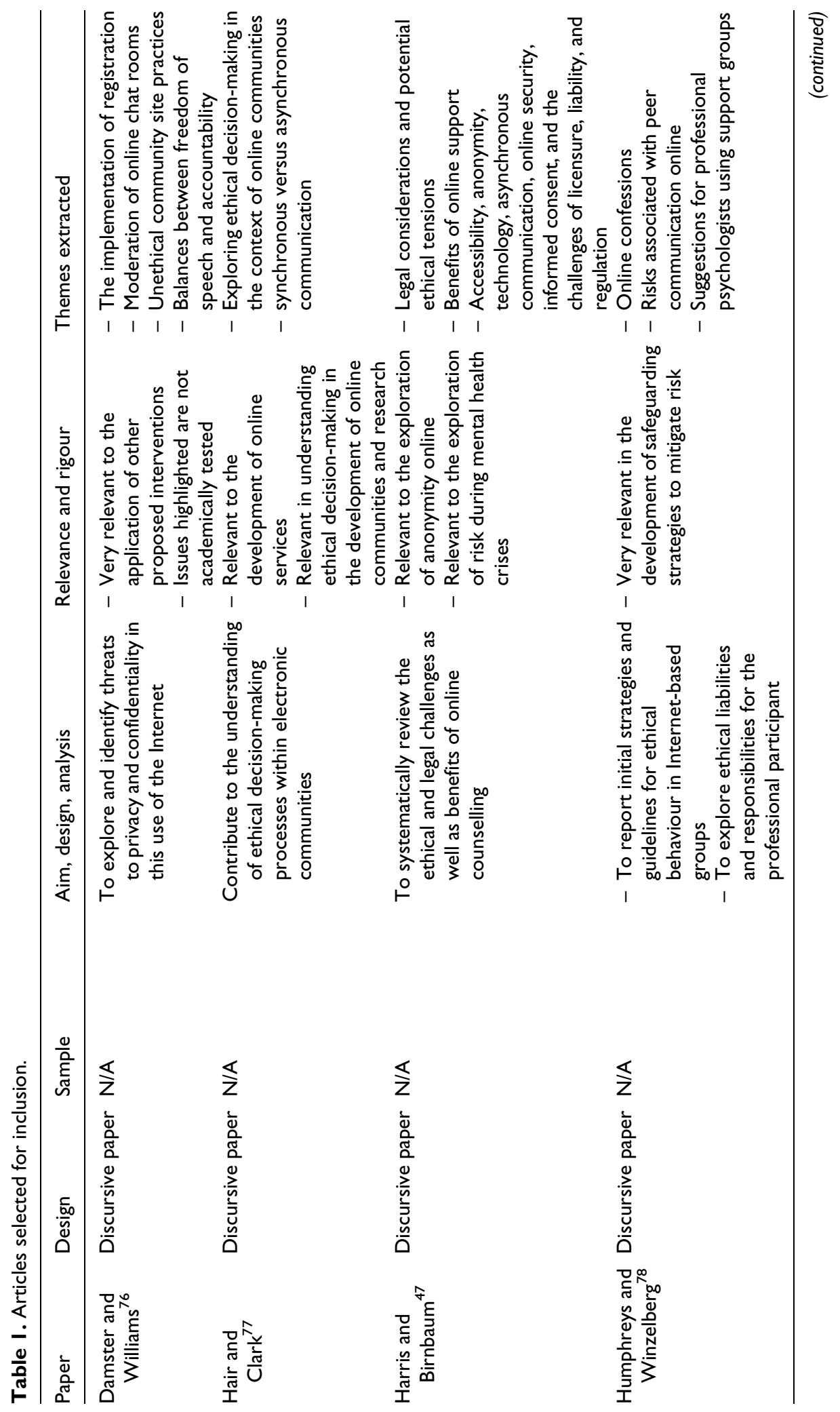




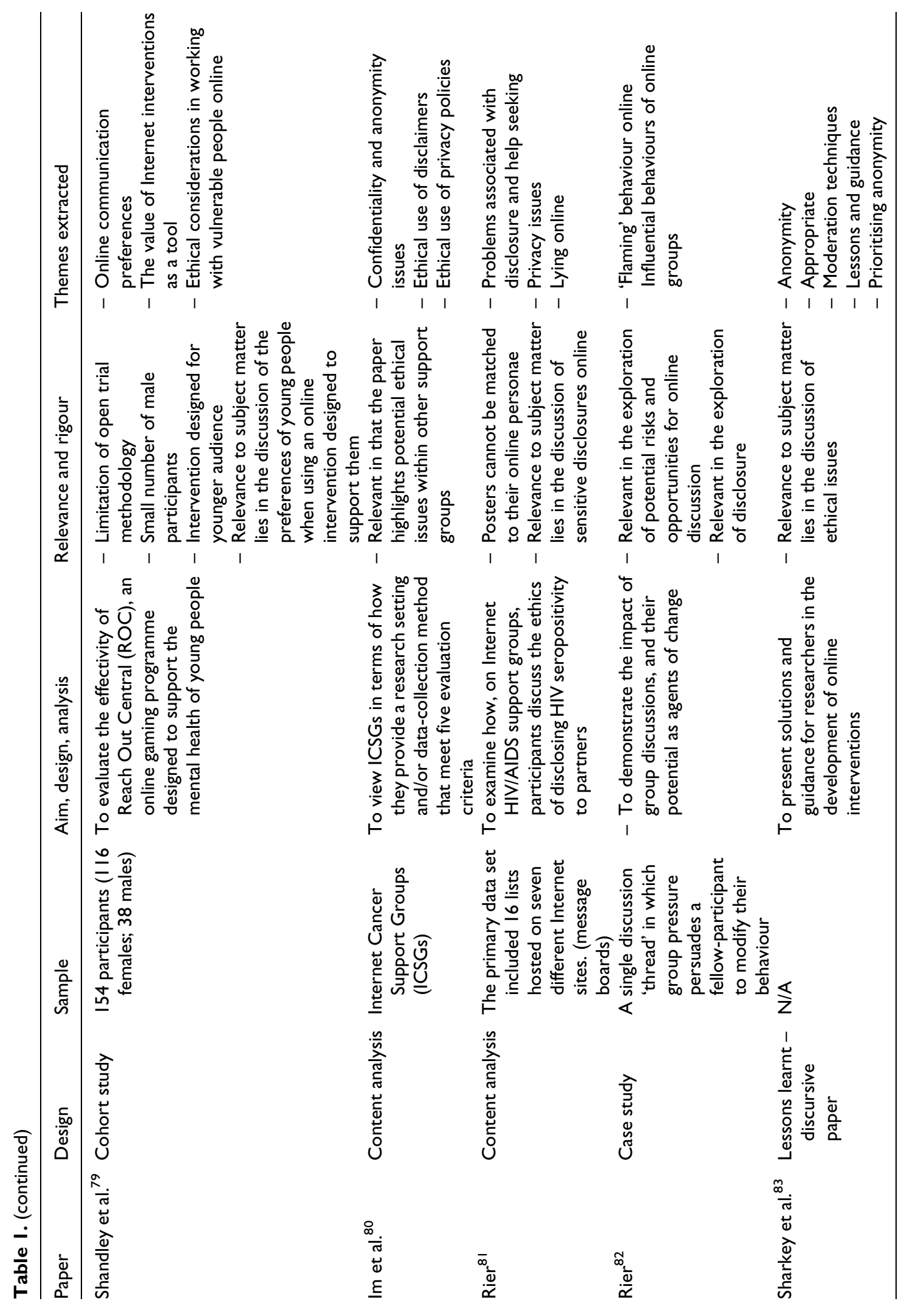




\section{Synthesis of findings}

To synthesise the data, any inferences or references to the key ethical themes of this review - confidentiality, anonymity and amnesty - were annotated through an iterative process of re-examination. As a number of papers retrieved did not describe their methodologies in great detail, data extraction remained limited to principle findings and theoretical concepts. This synthesis was guided by the realist synthesis methodology, where 'contradictory' evidence is used to generate insights about the influence of context. ${ }^{72}$

\section{Confidentiality}

Damster and Williams ${ }^{76}$ indicate that health professionals should be suspicious of any attempts to erode confidentiality, whether in the medical or other sectors, as it is worthy of protection, not just for the good of individuals, but also for the good of society as a whole. This discursive paper goes on to describe the medical ethics model, where a health professional will always strive to respect the confidentiality of information entrusted to [them] by the patient. In keeping with this model, they also state that it is the patient who has the right to decide who to share their information with, rather than the healthcare professional. Additionally, without the provision of confidentiality, Humphreys and colleagues assert that any ethical responsibilities associated with a psychotherapeutic relationship cannot be invoked. ${ }^{78}$

Hair and Clark ${ }^{77}$ explore the ethical challenges of preserving the confidentiality and anonymity of those engaging within virtual communities. They purport that a relatively rapid and synchronous form of communication such as one-to-one instant messaging may give a user an increased sense of confidentiality. Harris and Birnbaum ${ }^{47}$ systematically review the ethical and legal challenges of delivering therapies to vulnerable people online. Conversely, they highlight that asynchronous communication may enable deeper reflection, increasing self-awareness and self-expression. In any case, Humphreys and Winzelberg ${ }^{78}$ sug- $^{-}$ gest that all online users may at some point become confused as to which contributions may be confidential, group based, open or closed in nature.

Virtual communities value the free speech they uphold through the provision of confidentiality highly. ${ }^{76}$ Damster and Williams ${ }^{76}$ go on to report that however 'outrageous' this free speech may become, in the interest of maintaining a supportive online community, the moderation of discussions is seen by many as the preferred management option. Hair and Clark $^{77}$ maintain that users who choose to forfeit their own confidentiality must be made aware of any potential repercussions.

As Shandley et al. ${ }^{79}$ explore the efficacy of a youth-focused online intervention, they highlight that some young people may not access effective help because they fear that their confidentiality might be broken. They go on to share how an online intervention can effectively promote help seeking and support the health and wellbeing of younger people, especially when gamification techniques are employed. Within their online intervention, 'Reach Out Central', participants are encouraged to interact as they adopt the persona of a pre-determined character or avatar rather than exposing any real-world details about themselves. Each user or 'player' is assigned a coach to act as a guide and mentor as the user navigates their way through a series of interactions designed to remedy and explore episodes of psychological distress. Their results indicate that as young people engage with an online intervention in this way, they may experience a reduction in the use of maladaptive coping behaviours, increased resilience and adopt healthier coping behaviours. ${ }^{79}$

In learning lessons from a self-harm discussion forum study 'Sharp Talk', Sharkey et al. ${ }^{83}$ emphasise that vulnerable users of online interventions may desire confidentiality and anonymity as a condition of use. Within their protocols, they ensured that anything that may compromise a member's anonymity or confidentiality would be prohibited and removed accordingly. They also encouraged users to be known only by a chosen unique username or 'pseudonym' to ensure that confidentiality was maintained. ${ }^{83}$ In order to 
mitigate the risk of exposure in Internet-based groups, Humphreys and Winzelberg ${ }^{78}$ also propose that professionals, who access support groups in the role of a peer, should do so with the use of a pseudonym. Yet, when users of online interventions adopt pseudonyms or alternate identities as they converse within virtual communities, Damster and Williams ${ }^{76}$ assert that they may be unable to entirely hide behind either anonymity or confidentiality. This is because over time, users come to know one another and recognise and identify the behavioural patterns in those individuals who interact on a regular basis.

Through their feminist analysis of Internet Cancer Support Groups, Im et al. ${ }^{80}$ express concern that some online interventions fail to ensure and safeguard the confidentiality and anonymity of their members as they interact. Throughout their research, they remained keen not to impose upon the physical and psychological privacy of the support group members. In order to enforce confidentiality, Damster and Williams ${ }^{76}$ highlight the need to consider the implementation of disclaimers, privacy statements and guidance when looking to facilitate online interventions. It was identified by Im et al. ${ }^{80}$ that many websites use the terms 'site disclaimer' or 'privacy' to describe user information on 'confidentiality' issues. However, very few of these statements were aimed at preserving the confidentiality and anonymity of members. Instead, these statements tended to state that the online facilities were not to replace professional treatment and were to be used only for educational purposes. Throughout this feminist analysis of online support groups, only one site out of 546 was found to warn its users not to post anything of a confidential nature. ${ }^{80}$

Fundamentally, when confidentiality is assured by an online intervention, there are some immediate technical matters to consider. Harris and Birnbaum ${ }^{47}$ highlight the need to regularly update online security software, as the provision of online support remains an ever evolving field. They also describe how breaches in online security may occur, as unauthorised individuals intercept wireless signals and compromise what is thought to be confidential information. Hair and Clark $^{77}$ add that with the existence of search engines, archiving software and the retrieval of verbatim quotes, seemingly private and deleted posts may be recorded technically, without user knowledge.

The provision of online confidentiality also has practical implications where the collection and tracking of data would usually occur through the use of website 'cookies' and mailing lists. In this regard, Damster and Williams ${ }^{76}$ refer to the difficulties in obtaining consent for obtaining and sharing personal data without invading the provision of confidentiality. In order to address some of these ethical considerations, Sharkey, Humphreys and colleagues suggested that their participants created new email accounts upon joining the online community, as well as unique pseudonyms. ${ }^{78,83}$

\section{Anonymity}

Damster and Williams ${ }^{76}$ report that the Internet has a long-standing legacy and reputation for facilitating anonymity. Sharkey et al. ${ }^{83}$ concur with this statement, and report how young people who self-harm expect anonymity and enjoy its protective nature. Harris and Birnbaum ${ }^{47}$ also highlight the safety that anonymity can offer those seeking support, as it more readily allows for open and disinhibited disclosures. In this case, they suppose that an online intervention may be the safest place to discuss the most challenging and emotional issues. Yet, they also report that anonymity can encourage roleplay and misrepresentation. Damster and Williams ${ }^{76}$ agree by suggesting that anonymous communication can encourage verbal violence. Nevertheless, during a self-harm discussion forum study, Sharkey et al. ${ }^{83}$ stressed that without anonymity, online users of interventions can be reluctant to engage. As a result, this particular study rejected any alternatives to providing anonymity as discouraging to potential participants.

$\operatorname{Rier}^{81,82}$ explores the ethical dynamics of an HIV/AIDS online support group, and the moral suasions of its members through two content analyses. Anonymity is of great importance within this online support group, as group members often wanted to conceal the nature of their illness and, in some instances, their homosexuality. Face-to-face disclosures within this population are sometimes avoided, as disclosing their 
HIV status is often tantamount to admitting stigmatised behaviours or lifestyle choices. Within this online group, Rier ${ }^{82}$ describes how the group displayed an authentic mix of opinion, yet the most common position regarding disclosure ethics is full disclosure. As members of the group admit to disclosure avoidance, other members of the community make frequent and persuasive calls for disclosure. Ultimately, the provision of anonymity within this group enabled honest moral debates, open disclosures and personal reflections within the group. ${ }^{82}$

Reir $^{81}$ goes on to explore how these frequent calls for disclosure within the same HIV/AIDS online support group may translate into moral suasion within its community via a second content analysis of online group discussion. As in the example given, one member openly disclosed how they had been engaging frequently in unprotected sex without disclosing their HIV status. Following a series of comments which debated this as a moral issue, the member reflected upon their behaviour and decided to then disclose their acts and name those now at risk anonymously via their physician. The paper then goes on to highlight other instances where a group member is initially unsure about what to do but is willing to make anonymous disclosures online in order to seek advice. Some other individual members under scrutiny are described as initially offering resistance to the dominant discourse, but then eventually become prepared to declare realworld behaviour change either anonymously or otherwise, having been swayed by group discussion. ${ }^{81}$

Online anonymity is important for those who wish to conceal any individual circumstances or behaviours they consider to be shameful. ${ }^{82}$ Humphreys and Winzelberg ${ }^{78}$ recognise that healthcare professionals sometimes participate in Internet-based groups anonymously to address their own psychological and behavioural problems. Humphreys and Winzelberg ${ }^{78}$ recommend that the healthcare professional should maintain clear and consistent role definition as they switch between the roles of both therapist and casual member of the online community. Rier ${ }^{81}$ suggests that online participants can regard positive and moral persuasion as part of their ethical responsibilities, duty and function. Conversely, Sharkey et al. ${ }^{83}$ purport that those who are vulnerable online, may be at risk of coercion rather than positive influence. Hair and Clark $^{77}$ add that should names be associated with 'public' posts online, unsolicited contact and harassment may occur outside of the virtual community space. The use of pseudonyms is suggested in order to uphold ethical practice in this case.

When users refuse to disclosed misdeeds in a real-world context, flaming behaviours can also occur in protest to any perceived injustice online. ${ }^{82}$ In seeking a balance between anonymity and accountability in online discourse, Damster and Williams ${ }^{76}$ suggest a compromise of requiring users to initially register their identity with a moderator as they join the virtual community. Moderators may be health professionals or peer group members. The user may then choose to use their real name, or a pseudonym for any interactions they then make. In this case, anonymity remains a choice, and only the moderator can delete, report and remove inappropriate content or users. Additionally, Hair and Clark ${ }^{77}$ maintain that it must be decided whether the online community offers anonymity to all members, just primary posters, certain individuals or only those who respond to open posts.

Within the findings of an online forum study, Sharkey et al. ${ }^{83}$ reported that moderators were needed to ensure that anonymous online safety can be maintained, and a strong consensus that moderators ought to get involved in providing support. Contrary to this finding, Humphreys and Winzelberg ${ }^{78}$ recommend that health professionals should not imply a therapeutic relationship online, when the ethical responsibilities in doing so cannot be met, as may be the case where users remain anonymous online. In order to support online moderators in their task, Sharkey et al. ${ }^{83}$ suggest that online interventions issue forum rules and employ private messaging facilities, links to other online support, a discussion room for forum moderators and a 'report' button for users.

It was suggested by Humphreys and Winzelberg ${ }^{78}$ that, should an online intervention allow individuals to anonymously seek support, a potentially important avenue of assistance may be opened to professionals who need help but fear being identified. Yet, they also identify that concerns may arise where users 
remain anonymous in a time of crisis, as there lies a consequent inability to intervene. Nevertheless, some online interventions such as 'Sharp Talk', explored by Sharkey et al. ${ }^{83}$ have rejected the alternatives to total anonymity, as they have placed more value upon encouraged participation and the protective nature of anonymity in pursuit of a utilitarian approach to support. Yet should the focus of conversation turn towards suicidal thoughts, or self-harm, Sharkey et al. ${ }^{83}$ also highlight that this may increase the vulnerability of users.

Harris and Birnbaum ${ }^{47}$ assert that online interventions provide a natural and therapeutic sense of anonymity for users, and explore how this conflicts with the need to verify a user's identity. They go on to state that it is difficult, if not impossible, to acquire accurate and valid information on a user's identity, and question whether this acquisition may be of benefit to the user in any event. In the context of extreme risk and serious clinical issues, they also recognise the ethical obligations and duties of care in relation to the need to report those at risk for appropriate intervention. ${ }^{47}$ In these cases, they propose that face-to-face services may be more swift in providing immediate emergency care. Additionally, we are reminded that some methods of online support may not be able to express timely, and much needed, empathy to those in severe distress. In order to improve upon the lack of demonstrable empathy to those in distress online, the use of emoticons is suggested. ${ }^{47}$

Communication on the Internet can make issues of privacy, confidentiality and personal relationships confusing. ${ }^{78}$ When exploring the therapeutic properties of an online community, Damster and Williams ${ }^{76}$ highlight the conflicts between promoting the principles of anonymity and confidentiality, while also encouraging openness and freedom and ensuring the safety of participants. Harris and Birnbaum ${ }^{47}$ highlight the legal and ethical dilemmas where face-to-face contact remains absent and the provision of anonymity is upheld. They draw attention towards the inability to assert clinical judgement, gain informed consent, report accurate concerns in a timely manner and establish the mental or physical capacity of the user online.

Nevertheless, Harris and Birnbaum ${ }^{47}$ insist that online interventions must always conform to duty-toreport or duty-to-protect statutes. However, Humphreys and Winzelberg ${ }^{78}$ purport that because online users may come from a broad geographical area, it would be unlikely that any ethical responsibilities in the event of an emergency would be able to be executed completely in any case. In addition to this, Harris and Birnbaum assert that any statues may vary from place to place, and that the online user may reside in a separate jurisdiction to that of the online community. In order to mitigate risk, Harris and Birnbaum ${ }^{47}$ endorse the creation of emergency contact lists and details of supportive services within the user's community to enable swift self-referral to localised face-to-face support during emergencies.

\section{Amnesty}

Hair and Clark ${ }^{77}$ describe both confidentially and anonymity as the 'starting point' for defining themes to be interpreted as 'ethical canons' or 'codes'. Yet with total confidentiality and anonymity in place, their corollary, amnesty becomes inevitable. Within the retrieved literature, there were no explicit references to amnesties within online interventions. However, the concept of amnesty became implicit within some of the papers, as some described the importance of total anonymity and/or confidentiality. ${ }^{47,76,78,80,83}$

In the online discussion forums of an HIV/AIDS support group, one episode of amnesty is highlighted where an online user modifies their undesirable offline behaviour as a result of anonymous online disclosure. ${ }^{81}$ This was done with the understanding that there would be no negative consequences in doing so. In this case, the user experienced the support of the online community, the development of insight and a realworld behaviour change.

To illustrate how online interventions may present extreme ethical dilemmas, Humphreys and Winzelberg $^{78}$ describe a case study in which the father of a 5-year-old girl confesses to her murder within an online support group. Within this scenario, some members of the community reported the crime to the authorities, 
and yet the healthcare professionals involved did not. In effect, the healthcare professionals respected the confidentiality of the disclosure and afforded the perpetrator amnesty. This ignited debate as to what the purpose, roles and responsibilities of an online support group may be, although no conclusions are presented in this case.

Some users within online communities have been seen to assume the role of a moral agent, and attempt to influence fellow users to exercise 'responsibility' by disclosing and acting upon their compromising predicaments to the appropriate authorities. ${ }^{82}$ In this sense, users of an online intervention look to guide both the online and offline behaviours of other users in order to achieve the most desirable outcome. Rier highlights these episodes during online egalitarian moral debates, where an inherent amnesty enabled those in distress to be persuaded to 'do the right thing' while maintaining a private identity. Rier ${ }^{82}$ concludes by suggesting that such online communities are a mechanism for engaging in support and moral suasion, where users both seek help and to enforce what the community defines as 'ethical conduct' within a real-world scenario.

\section{Discussion}

\section{Summary of findings}

This realist review has identified nine papers that explore key themes of confidentiality, anonymity and amnesty in relation to online interventions designed to provide support. Findings suggest that confidentiality, anonymity, and their corollary, amnesty, are important in the optimisation of open disclosure, trust, real-world behaviour change, engagement and help-seeking online. These findings can also be mapped against the pathway to disclosures model, where anonymous participation online can lead to open disclosures and help-seeking offline. ${ }^{23}$ However, ethical dilemmas remain where there is a legal duty to report, disclose and act upon concerns which may put both the online user and the public at risk. Ethical considerations were also highlighted, as obligations to ensure that appropriate and real-world care is given to the online user may not be met, should both anonymity and confidentiality be guaranteed in full.

Through this review, we find that there are a range of ethical considerations to consider in the development of online interventions to support midwives. In order to develop insights into the influence of context, these findings must be mapped against the ethical and legal considerations pertaining to midwives in distress.

\section{Strengths and limitations}

Due to this being a theory-driven approach, this realist methodology enabled the researchers to make use of any 'grey literature' rather than relying solely upon formal research in the exploration of complex ethical considerations. This literature has the ability to add to the synthesis, often providing contextual information which would otherwise be omitted.

The realist synthesis review methodology employs iterative searching techniques in favour of systematic searches. We recognise this as a limitation because such searches cannot be replicated. However, because searching is initially broad in scope and is refined through progressive focusing, this review was also able to respond flexibly to new findings as they emerged. ${ }^{72}$

The search terms, selection procedures and processes of analysis prescribed by the realist review methodology also favour a flexible and unsystematic approach. Although this enables a direct approach to synthesising the literature, we also recognise that significant papers may have avoided retrieval.

Unfortunately, this review did not retrieve any papers that directly addressed the subject of midwives using online interventions; therefore, it has been necessary to extrapolate from other groups to midwives. 


\section{Legal and ethical considerations associated with online interventions}

Developers of online interventions designed to support those in distress can follow the e-Health Code of Ethics, which ensures that people worldwide can confidently and with full understanding of the known risks realise the potential of the Internet in managing their own health and the health of those in their care. ${ }^{84}$ However, this guidance does not cover the development of unique online sources for the provision of support to healthcare professionals. ${ }^{85}$ Midwives in the United Kingdom must maintain public confidence in the nursing professions and uphold standards and professional behaviour. ${ }^{86}$ These midwives have a duty to escalate any professional concerns pertaining to both themselves and their colleagues, yet if a concern arises within an online platform, a midwife may be left unable to identify the perpetrator or escalate concerns.

Midwives in the United Kingdom are duty bound to ensure that any support that they give to colleagues must not compromise or be at the expense of patient or public safety. ${ }^{86}$ Midwives in distress may disclose episodes of impairment, medical error or display unprofessional behaviour within an online intervention designed to support them. These episodes of impairment may put patients at immediate risk of harm and may ordinarily prompt a referral to the regulators and further investigation for the immediate protection of the public. Yet, the issues highlighted here may prompt the question whether a midwife in distress has the same rights to confidentiality as the 'typical' online user in distress.

Midwives who seek out an online platform for support may be psychologically vulnerable. It has been argued that those providing online therapies should know the location and identity of those users at risk of suicide in the event of a psychological emergency. ${ }^{87}$ This may not be possible for an online intervention offering total anonymity to its users. Despite this, it has also been argued that the benefits of providing online therapies far outweigh these risks. ${ }^{88}$ Moreover, the challenge to locate a suicidal online user has been found to be no more difficult than locating an 'at risk' individual engaging with telephone therapy. ${ }^{89}$ As such, in signposting the anonymous midwives who engage with an online platform towards outside sources of support, an online intervention may offer a portal for knowledge exchange and on-going care in the absence of immediate professional support.

Although the literature rarely highlights the legal considerations of providing support via online interventions, we recognise that midwives currently have a legal obligation and duty of care to maintain confidentiality and report concerns in line with their professional codes of conduct for the protection of the public. However, in the context of online interventions, the legal regulations that apply to online clinician-patient interaction may mean that the dissemination of concerns to any third party becomes prohibited. ${ }^{90}$ Additionally, as Internet access becomes global, users and facilitators will need to consider their legal jurisdiction and authority to practice in areas beyond both their professional or geographical territory.

In relying on the process of moral peer review and culture setting, online interventions may sacrifice immediate public protection in pursuit of wider and more sustainable advances in public safety and protection. Additionally, it is of note that anonymity may become less appropriate for serious cases, where there may be an ethical obligation of duty to report a user for further intervention. In these cases, we are reminded of the requirement to follow duty to report and protect statutes. These questions, related to jurisdictional challenges may require further dialogue with professional associations and regulatory bodies. ${ }^{47}$

Legal and ethical issues endure where there remains an inability to assert clinical judgement, gain informed consent and establish mental capacity while users remain unidentifiable online. ${ }^{47}$ In order to address legal and ethical considerations, some online interventions have used disclaimers and privacy statements as a means of either protecting the intervention against its own accountabilities or to instruct its users upon how they may or may not expect their privacy to be upheld. ${ }^{80}$ Legal obligations vary geographically and nationally, from one country to the next. In England, for example, the law is the same 
whether you work in the south of England or the north of England, yet in many states of America, there may be conflicting legal obligations in force. In this context, a global online intervention for midwives could establish its own codes of conduct and level of accountability, guided by the level of accountability set by regulators around the world.

Facilitators of an online intervention designed to support midwives could be specialist healthcare professionals or individual midwives proficient in restorative supervision and peer support. However, these professionals would still be legally obligated to report impaired midwives to their regulatory body. As such, strong privacy statements and usage policy agreements may be required.

\section{Confidentiality, anonymity and amnesty}

Online interventions, which promote the principles of both anonymity and confidentiality, also permit their corollary, amnesty. If online interventions were adapted to support midwives in psychological distress, there lies the risk of non-disclosure of poor clinical practice, as midwives may look to seek anonymous support in order to avoid accountability. Without being able to identify the users of an online intervention, no real-world interventions, referrals or accountability can reliably be pursued. Therefore, it may be that society is only willing to permit an amnesty in the cases of relatively trivial matters, rather than in severe cases. However, any attempt to measure the degrees of severity may result in some episodes not being perceived as objectively severe in nature.

For an online intervention to support midwives, it will be important to decide which control measures should be employed to discourage undesirable behaviours such as those which may undermine public confidence in the profession, bullying or 'flaming'. The online inhibition effect in these cases can be toxic. ${ }^{45}$ Other online communities hold a 'real name' policy in order to hold users to account; however, these have previously led to nontrivial, on-going disputes, which may inhibit the development of productive online communities. ${ }^{91}$ In this case, midwives who are reluctant to speak openly may not engage with an intervention where they may be further held to account.

Moderators of online support groups have noted that trust in confidentiality and anonymity is an essential part of maintaining a successful health-related online support group. ${ }^{92-94}$ The provision of anonymity and confidentiality may also appeal to those who would ordinarily feel unable to disclose a sensitive issue. As confidentiality and anonymity have been cited as two of the most important features of an online peer support forum, these two principles may be key features in online interventions to support midwives in work-related psychological distress. ${ }^{95}$ In order to mitigate risk, users may require ethical guidance in relation to the maintenance of confidentiality in the context of any work-related discussions.

When a user is grappling with a moral issue, they may be more likely to disclose in an online environment that allows for anonymity for the purpose of help seeking. In an online environment, where morality can be debated, users can also be persuaded by the community to modify their behaviours and eventually make real-world disclosures. In this context, an online intervention may have the ability to change any reticent behaviour seen in some midwives, which would in turn aid help seeking and increase public protection. As such, the serious risks involved with the provision of amnesty online may be mitigated somewhat by the possibility of encouraging a larger number of midwives to seek help, modify any risky behaviours and move towards a real-world disclosure and self-referral in line with the pathways to disclosure model. ${ }^{23}$

\section{Ethical decision-making}

Ethicists are largely concerned with doing right, following the principles of justice, beneficence through identifying risk, and preventing harm through protecting privacy, being honest, obtaining consent and 
respecting a person's inherent value as a human being. ${ }^{77}$ Ethical decision-making within the creation of electronic communities can be derived from two main philosophical approaches. These have been described by Hair and Clark ${ }^{77}$ as deontology, which is focussed upon using codes of conduct in decision-making, and teleontology, which advocates achieving the greatest good for the greatest number of people. As such, hosts of online communities must balance the effects upon the entire community with the individual risks that may $^{\text {arise. }^{77}}$

It has been suggested that individuals progress through three different levels as they make moral judgements: (a) the pre-conventional level, when moral decisions are based on rewards and punishments and obedience to authority; (b) the conventional level, when individuals recognise societal laws and rules and are concerned regarding collective welfare and (c) the post-conventional level, when moral decisions are based on internalised moral values and abstract principles. ${ }^{96}$ At the peak stage of moral decision development, a concern for wider social justice and human rights becomes evident. ${ }^{96}$

Ethical dilemmas such as those presented within this article are often complex and ambiguous. Many ethical decision-making frameworks exist to assist nursing populations in making ethical choices. ${ }^{97}$ These often focus upon the alleviation of suffering, responsibilities to the public and professional accountability, where the nurse or midwife's primary commitment is to the patient. Midwives who use an online intervention could be analogous to patient users if the work of Damster and Williams ${ }^{76}$ is applied to the present issue. In any case, within these ethical frameworks, there is also a focus on personal health and wellbeing, collegial support, competency maintenance and professional growth, as it is widely recognised that both patients and the public are safest while nurses and midwives remain in optimal mental and physical health.

Generally, ethical decision-making within the nursing professions leans towards a favourable riskbenefit ratio. ${ }^{97,98}$ Teleological approaches focus upon the final effects of human action. ${ }^{99}$ Conversely, the wider philosophical approach of utilitarianism is founded upon the premise that an action is ethical if the outcomes of the action lead to the greatest benefits for society at large with the fewest possible negative consequences. ${ }^{100}$ In this context, society may gain the greatest benefit from supporting the midwifery workforce to remain psychologically safe. Yet, if midwives are to be supported via an online intervention, society may also have to accept that midwives need confidentiality, anonymity and amnesty to do so.

\section{Conclusion and recommendations}

Many practitioner health programmes exist to support physicians and afford those in distress identity privacy for the purpose of help seeking and recovery. ${ }^{66,101,102}$ Midwives, along with other healthcare professionals, may require specialist support among their own kind, away from other health service users. ${ }^{66,103}$ This specialist support may need to embody the principles of confidentiality, anonymity and a resulting amnesty. This realist synthesis review has considered these principles in the context of online interventions to support midwives in work-related psychological distress.

The principles of confidentiality, anonymity and amnesty online may appeal to midwives in workrelated psychological distress who feel stigmatised, are pressured for time, fear retribution and/or frequently access the Internet. ${ }^{12,104,105}$ However, in deciding whether this provision may be ethically justifiable, online intervention providers must weigh up the risk/benefit ratio to both patients, midwives and the wider general public. ${ }^{106}$ We have discussed and characterised the most morally justifiable and ethical decision from a utilitarian perspective as, the greatest good for the greatest number. ${ }^{107}$

Online interventions may offer an opportunity to improve the help-seeking behaviours, rates of disclosure and provision of therapeutic support of midwifery populations when they allow for confidentiality, anonymity and amnesty. ${ }^{59,108-110}$ The consequences of failing to adequately support midwives in workrelated psychological distress may mean that our maternity services experience a less compassionate workforce, reduced productivity, reduced standards of care and increased rates of error. ${ }^{7,12,11-113}$ As such, 
we argue that the morally justifiable decision may be to provide an opportunity for midwives to privately manage their emotional fears, improve their emotional well-being, optimism, mental health literacy and openly engage with emotional support via an online intervention, as this may outweigh any potentially damaging processes. ${ }^{114}$ Additionally, midwives are entitled to a psychologically safe professional journey as they work in equal partnership with childbearing women.

International codes of conduct promote that midwives should 'support and sustain each other in their professional roles, and actively nurture their own and others' sense of self-worth'. ${ }^{115}$ The Nursing and Midwifery Council ${ }^{86}$ also recognise the importance of the need for their registrants to 'be supportive of colleagues who are encountering health or performance problems'. Yet, the caveat associated with this support is that it must never compromise or be at the expense of patient or public safety. We argue that in effectively supporting midwives anonymously online, we may be able to protect both patients and the public via more sustainable means. As such, the benefits of allowing anonymous free speech for the purpose of supporting midwives in distress may outweigh the need for the immediate identification and reporting of episodes of impairment for the purpose of instant accountability.

The risks associated with providing online interventions to support midwives in psychological distress may be somewhat mitigated by the ethos of the support group, which may preclude confrontations' over risky and/or immoral behaviour. ${ }^{116}$ Users may also embrace a collective philosophy that promotes adages such as, 'honesty is the best policy' and 'do unto others'. ${ }^{82}$ Therefore, in influencing positive group behaviours, midwives may exercise their own responsibilities to disclose issues to regulatory bodies where appropriate with the support of others in line with the pathways to disclosure model. ${ }^{23} \mathrm{We}$ consider this to be the preferred outcome for online support interventions, where midwives receive support and yet moral accountability is respected.

Additionally, we consider that in line with other populations accessing online interventions for support and practical advice, midwives may not necessarily reject their existing moral frameworks at the same time. ${ }^{82}$ Therefore, the morally justifiable and ethical decision, promoting the greatest good for the greatest number may be to permit anonymity, confidentiality and amnesty in pursuit of healthier midwives for safer maternity services overall.

This article has explored the ethical, legal and moral issues associated with online interventions to support midwives in work-related psychological distress. Although we argue that the principles of confidentiality, anonymity and amnesty should be upheld in the pursuit of the greatest benefit for the greatest number of people, we also call for a further dialogue in relation to this matter in pursuit of robust ethical stability.

\section{Acknowledgements}

The authors would like to thank anonymous peer reviewers for helping to shape and strengthen this paper. The views expressed within this submitted article are the authors own, and not an official position of any other institution or funder.

\section{Declaration of Conflicting Interests}

The author(s) declared no potential conflicts of interest with respect to the research, authorship and/or publication of this article.

\section{Funding}

The author(s) disclosed receipt of the following financial support for the research, authorship, and/or publication of this article: This study has been funded via a full-time $\mathrm{PhD}$ scholarship provided by Coventry University. 


\section{References}

1. Dixon-Woods M, Baker R, Charles K, et al. Culture and behaviour in the English National Health Service: overview of lessons from a large multimethod study. BMJ Qual Saf 2014; 23: 106-115.

2. Wilkinson E. UK NHS staff: stressed, exhausted, burnt out. Lancet 2015; 385: 841-842.

3. Sull A, Harland N and Moore A. Resilience of health-care workers in the UK; a cross-sectional survey. J Occup Med Toxicol 2015; 10: 20.

4. Moll S, Patten SB, Stuart H, et al. Beyond silence: protocol for a randomized parallel-group trial comparing two approaches to workplace mental health education for healthcare employees. BMC Med Educ 2015; 15: 78.

5. Trippany RL, Kress VEW and Wilcoxon SA. Preventing vicarious trauma: what counselors should know when working with trauma survivors. J Couns Dev 2004; 82: 31-37.

6. Van Voorhees BW, Fogel J, Houston TK, et al. Attitudes and illness factors associated with low perceived need for depression treatment among young adults. Soc Psychiatry Psychiatr Epidemiol 2006; 41: 746-754.

7. The Royal College of Physicians. Work and wellbeing in the NHS: why staff health matters to patient care. London: The Royal College of Physicians, 2015.

8. Cumberlege J. BETTER BIRTHS - improving outcomes of maternity services in England: a five year forward view for maternity care, https://www.england.nhs.uk/wp-content/uploads/2016/02/national-maternity-reviewreport.pdf

9. Young CM, Smythe L and Couper JM. Burnout: lessons from the lived experience of case loading midwives. Int $J$ Childbirth 2015; 5: 154-165.

10. Robins K. Psychological trauma of nurse-midwives following shoulder dystocia complicated by neonatal morbidity or mortality. Portland, OR: Oregon Health \& Science University, 2012.

11. Sheen K, Spiby H and Slade P. The experience and impact of traumatic perinatal event experiences in midwives: a qualitative investigation. Int J Nurs Stud 2016; 53: 61-72.

12. Currie L and Richens Y. Exploring the perceptions of midwifery staff about safety culture. Br J Midwifery 2009; 17: 783-790.

13. Chang T. Online counseling: prioritizing psychoeducation, self-help, and mutual help for counseling psychology research and practice. Couns Psychol 2005; 33: 881-890.

14. Suler J. The online disinhibition effect. Cyberpsychol Behav 2004; 7: 321-326.

15. Beauchamp TL and Childress JF. Principles of biomedical ethics. Oxford: Oxford University Press, 2001.

16. Jones C. The utilitarian argument for medical confidentiality: a pilot study of patients' views. J Med Ethics 2003; 29: $348-352$.

17. Kipnis K. A defense of unqualified medical confidentiality. Am J Bioeth 2006; 6: 7-18.

18. Saunders B, Kitzinger J and Kitzinger C. Participant anonymity in the Internet age: from theory to practice. Qual Res Psychol 2015; 12: 125-137.

19. Kirsten A. GUNS UNDER FIRE: initial results of the 2005 firearms amnesty. SA Crime Quart, https://www. issafrica.org/uploads/KIRSTEN.PDF

20. Kenyon SL, Ramsey JD, Lee T, et al. Analysis for identification in amnesty bin samples from dance venues. Ther Drug Monit 2005; 27: 793-798.

21. Eades C. Young people and knife carrying: what to do? Childright 2006; 231: 10-13.

22. West LM, Diack L, Cordina M, et al. A systematic review of the literature on 'medication wastage': an exploration of causative factors and effect of interventions. Int J Clin Pharm 2014; 36: 873-881.

23. Cooper G. Exploring and understanding online assistance for problem gamblers: the pathways disclosure model. Int J Ment Health Addict 2004; 1: 32-38.

24. Cooper GA. Online assistance for problem gamblers: an examination of participant characteristics and the role of stigma. Toronto, ON, Canada: University of Toronto, 2001.

25. Boorman S. Health and well-being of the NHS workforce. J Public Ment Health 2010; 9: 4-7. 
26. NHS EmployersReducing sickness absence in the NHS using evidence-based strategies. Summary report, 2014, http://www.nhsemployers.org/ /media/Employers/Publications/Reducing\%20sickness\%20absence\%20in \%20the\%20NHS.pdf

27. Pezaro S, Clyne W, Turner A, et al. 'Midwives overboard!' Inside their hearts are breaking, their makeup may be flaking but their smile still stays on. Women Birth. Epub ahead of print 27 October 2015. DOI: 10.1016/j.wombi. 2015.10.006.

28. Aitamaa E, Leino-Kilpi H, Iltanen S, et al. Ethical problems in nursing management: the views of nurse managers. Nurs Ethics. Epub ahead of print 21 April 2015. DOI: 10.1177/0969733015579309.

29. Leinweber J and Rowe HJ. The costs of 'being with the woman': secondary traumatic stress in midwifery. Midwifery 2010; 26: 76-87.

30. Rice $\mathrm{H}$ and Warland J. Bearing witness: midwives experiences of witnessing traumatic birth. Midwifery 2013; 29 : $1056-1063$.

31. Sheen K, Slade P and Spiby H. An integrative review of the impact of indirect trauma exposure in health professionals and potential issues of salience for midwives. $J$ Adv Nurs 2014; 70: 729-743.

32. Muliira RS and Bezuidenhout MC. Occupational exposure to maternal death: psychological outcomes and coping methods used by midwives working in rural areas. Midwifery 2015; 31: 184-190.

33. Austin D, Smythe E and Jull A. Midwives' well-being following adverse events - what does the research indicate? New Zealand Coll Midwives J 2014; 50: 19-23.

34. Seys D, Scott S, Wu A, et al. Supporting involved health care professionals (second victims) following an adverse health event: a literature review. Int J Nurs Stud 2013; 50: 678-687.

35. Ullström S, Andreen Sachs M, Hansson J, et al. Suffering in silence: a qualitative study of second victims of adverse events. BMJ Qual Saf 2014; 23: 325-331.

36. Hildingsson I and Fenwick J. Swedish midwives' perception of their practice environment - a cross sectional study. Sex Reprod Healthc 2015; 6: 174-181.

37. Hunter B and Warren L. Midwives' experiences of workplace resilience. Midwifery 2014; 30: 926-934.

38. Denham CR. TRUST: the 5 rights of the second victim. J Patient Saf 2007; 3: 107-119.

39. Kirkham M and Stapleton H. Midwives' support needs as childbirth changes. $J$ Adv Nurs 2000; 32: 465-472.

40. Calvert I. Support for midwives - a model of professional supervision based on the recertification programme for midwives in New Zealand. Women Birth 2014; 27: 145-150.

41. Kirkham M. The culture of midwifery in the National Health Service in England. J Adv Nurs 1999; 30: 732-739.

42. Ziebland S and Wyke S. Health and illness in a connected world: how might sharing experiences on the Internet affect people's health? Milbank $Q$ 2012; 90: 219-249.

43. Barak A, Hen L, Boniel-Nissim M, et al. A comprehensive review and a meta-analysis of the effectiveness of Internet-based psychotherapeutic interventions. J Technol Hum Serv 2008; 26: 109-160.

44. Kuester A, Niemeyer $\mathrm{H}$ and Knaevelsrud C. Internet-based interventions for posttraumatic stress: a meta-analysis of randomized controlled trials. Clin Psychol Rev 2016; 43: 1-16.

45. Allen C, Vassilev I, Kennedy A, et al. Long-term condition self-management support in online communities: a meta-synthesis of qualitative papers. J Med Internet Res 2016; 18: e61.

46. Preece J. Online communities: designing usability and supporting sociability. Hoboken, NJ: John Wiley \& Sons, 2000.

47. Harris B and Birnbaum R. Ethical and legal implications on the use of technology in counselling. Clin Soc Work J 2015; 43: 133-141.

48. Ellenchild WJ. Confidentiality: concept analysis and clinical application. In: Yoder-Wise PS (ed.) Nursing forum. Hoboken, NJ: Wiley Online Library, 2000, pp. 5-16.

49. Kerkhof AJ, Van Spijker BA and Mokkenstorm JK. Reducing the burden of suicidal thoughts through online cognitive behavioural therapy self help. In: Mishara B and Kerkhof A (eds) Suicide prevention and new technologies: evidence based practice. Basingstoke: Palgrave Macmillan, 2013, pp. 50-62. 
50. Carretta CM, Burgess AW and DeMarco R. To tell or not to tell. Violence Against Women 2015; 21: 1145-1165.

51. Santor DA, Poulin C, LeBlanc JC, et al. Online health promotion, early identification of difficulties, and help seeking in young people. $J$ Am Acad Child Adolesc Psychiatry 2007; 46: 50-59.

52. Gulliver A, Griffiths KM, Christensen H, et al. A systematic review of help-seeking interventions for depression, anxiety and general psychological distress. BMC Psychiatry 2012; 12: 81.

53. King R, Bambling M, Lloyd C, et al. Online counselling: the motives and experiences of young people who choose the Internet instead of face to face or telephone counselling. Counsell Psychother Res J 2006; 6: $169-174$.

54. Dunlop SM, More E and Romer D. Where do youth learn about suicides on the Internet, and what influence does this have on suicidal ideation? J Child Psychol Psychiatry 2011; 52: 1073-1080.

55. Williams SG. The ethics of Internet research. Online J Nurs Inform 2012; 16: 38-48.

56. Burkell J. Anonymity in behavioural research: not being unnamed, but being unknown. Univ Ottawa Law Technol $J$ 2006; 3: 189-203.

57. Kambourakis G. Anonymity and closely related terms in the cyberspace: an analysis by example. J Inform Secur Appl 2014; 19: 2-17.

58. Calvert I and Benn C. Trauma and the effects on the midwife. Int J Childbirth 2015; 5: 100-112.

59. Crisp DA and Griffiths KM. Participating in online mental health interventions: who is most likely to sign up and why? Depress Res Treat 2014; 2014: 1-11.

60. Hook A and Andrews B. The relationship of non-disclosure in therapy to shame and depression. Br J Clin Psychol 2005; 44: 425-438.

61. Smyth JM, Pennebaker JW and Arigo D. Chapter 8: what are the health effects of disclosure? In: Revenson TA and Singer JE (eds) Handbook of health psychology. Abingdon: Psychology Press, 2012, pp. 175-192.

62. Krautscheid LC. Defining professional nursing accountability: a literature review. J Prof Nurs 2014; 30: $43-47$.

63. Weisman N. A history and discussion of amnesty. Columbia Hum R Law R 1972; 4: 529.

64. Brush BL, McGee EM, Cavanagh B, et al. Forgiveness: a concept analysis. $J$ Holist Nurs 2001; 19: $27-41$.

65. Moberly T. GMC is 'traumatising' unwell doctors and may be undermining patient safety, Gerada says. $\mathrm{Br}$ Med J Career, 2014, http://careers.bmj.com/careers/advice/view-article.html?id=20017662

66. Brooks SK, Gerada C and Chalder T. Doctors and dentists with mental ill health and addictions: outcomes of treatment from the Practitioner Health Programme. J Ment Health 2013; 22: 237-245.

67. Cohen D, Winstanley SJ and Greene G. Understanding doctors' attitudes towards self-disclosure of mental ill health. Occup Med. Epub ahead of print 29 March 2016. DOI: 10.1093/occmed/kqw024.

68. BMA Medical Ethics. Ethical responsibilities in treating doctors who are patients: guidance from the BMA Medical Ethics Department, http://www.gmc-uk.org/doctorswhoarepatientsjanuary2010.pdf_62126868.pdf

69. Radhakrishna S. Culture of blame in the National Health Service; consequences and solutions. Br J Anaesth 2015; 115: 653-655.

70. Shim M, Cappella JN and Han JY. How does insightful and emotional disclosure bring potential health benefits? Study based on online support groups for women with breast cancer. J Commun 2011; 61: 432-454.

71. Traynor M, Stone K, Cook H, et al. Disciplinary processes and the management of poor performance among UK nurses: bad apple or systemic failure? A scoping study. Nurs Inq 2014; 21: 51-58.

72. Pawson R, Greenhalgh T, Harvey G, et al. Realist review - a new method of systematic review designed for complex policy interventions. J Health Serv Res Policy 2005; 10(Suppl. 1): 21-34.

73. Wong G, Greenhalgh T, Westhorp G, et al. RAMESES publication standards: realist syntheses. BMC Med 2013; 11: 21 .

74. Marchal B, Van Belle S, Van Olmen J, et al. Is realist evaluation keeping its promise? A review of published empirical studies in the field of health systems research. Evaluation 2012; 18: 192-212.

75. Pawson R, Greenhalgh T, Harvey G, et al. Realist synthesis: an introduction. Manchester: ESRC Research Methods Programme, University of Manchester, 2004. 
76. Damster G and Williams JR. The Internet, virtual communities and threats to confidentiality. S Afr Med J 1999; 89: $1175-1178$.

77. Hair $\mathrm{N}$ and Clark M. The ethical dilemmas and challenges of ethnographic research in electronic communities. Int J Market Res 2007; 49: 781-800.

78. Humphreys K and Winzelberg A. Psychologists' ethical responsibilities in Internet-based groups: issues, strategies, and a call for dialogue. Prof Psychol Res Pr 2000; 31: 493-496.

79. Shandley K, Austin D, Klein B, et al. An evaluation of 'Reach out central': an online gaming program for supporting the mental health of young people. Health Educ Res 2010; 25: 563-574.

80. Im E, Chee W, Tsai H, et al. Internet cancer support groups: a feminist analysis. Cancer Nurs 2005; 28: 1-7.

81. Rier DA. The impact of moral suasion on Internet HIV/AIDS support groups: evidence from a discussion of seropositivity disclosure ethics. Health Sociol Rev 2007; 16: 237-247.

82. Rier DA. Internet social support groups as moral agents: the ethical dynamics of HIV+ status disclosure. Sociol Health Illn 2007; 29: 1043-1058.

83. Sharkey S, Jones R, Smithson J, et al. Ethical practice in Internet research involving vulnerable people: lessons from a self-harm discussion forum study (SharpTalk). J Med Ethics 2011; 37: 752-758.

84. e-Health Ethics Initiative. e-Health code of ethics (May 24). J Med Internet Res 2000; 2: e9.

85. Vayena E, Mastroianni A and Kahn J. Ethical issues in health research with novel online sources. Am J Public Health 2012; 102: 2225-2230.

86. The Nursing and Midwifery Council (NMC). The code: professional standards of practice and behaviour for nurses and midwives. London: NMC, 2015.

87. Rummell CM and Joyce NR. 'So wat do u want to wrk on 2day?': The ethical implications of online counseling. Ethics Behav 2010; 20: 482-496.

88. Ravis H. Challenges and special problems in distance counseling: how to respond to them. In: Malone JF, Miller RM and Walz GR (eds) Distance counseling: expanding the counselor's reach and impact. Alexandria, VA: American Counseling Association, 2007, pp. 119-132.

89. Rochlen AB, Zack JS and Speyer C. Online therapy: review of relevant definitions, debates, and current empirical support. J Clin Psychol 2004; 60: 269-283.

90. Dever Fitzgerald T, Hunter PV, Hadjistavropoulos T, et al. Ethical and legal considerations for Internet-based psychotherapy. Cogn Behav Ther 2010; 39: 173-187.

91. Cho D and Kwon KH. The impacts of identity verification and disclosure of social cues on flaming in online user comments. Comput Hum Behav 2015; 51: 363-372.

92. Frost J, Vermeulen IE and Beekers N. Anonymity versus privacy: selective information sharing in online cancer communities. J Med Internet Res 2014; 16: e126.

93. Kauer SD, Mangan C and Sanci L. Do online mental health services improve help-seeking for young people? A systematic review. J Med Internet Res 2014; 16: e66.

94. Coulson NS and Shaw RL. Nurturing health-related online support groups: exploring the experiences of patient moderators. Comput Hum Behav 2013; 29: 1695-1701.

95. Horgan A, McCarthy G and Sweeney J. An evaluation of an online peer support forum for university students with depressive symptoms. Arch Psychiatr Nurs 2013; 27: 84-89.

96. Kohlberg L. The philosophy of moral development: moral stages and the idea of justice. New York: Harper \& Row, 1981.

97. Mallari M, Grace M and Joseph D. Ethical frameworks for decision-making in nursing practice and research: an integrative review, 2016, https://works.bepress.com/jdtariman/22/

98. Peter E. The interplay between the abstract and the particular: research ethics standards and the practice of research as symbolic. Nurs Sci $Q$ 2006; 19: 20-24; discussion 19.

99. Noble D. The conceptualist view of teleology. Analysis 1967; 28: 62-63.

100. Beuachamp T and Bowie NE. Ethical theory and business. Englewood Cliffs, NJ: Prentice Hall, 1983. 
101. Maruthappu M, Sood H and Black C. Prioritising prevention and the health of NHS staff. Lancet 2015; 386 : $1322-1323$.

102. Skipper GE and DuPont RL. The physician health program: a replicable model of sustained recovery management. In: Kelly JF and White WL (eds) Addiction recovery management. New York: Humana Press, 2010, p. 281.

103. Brooks SK, Gerada $\mathrm{C}$ and Chalder $\mathrm{T}$. Review of literature on the mental health of doctors: are specialist services needed? J Ment Health 2011; 20: 146-156.

104. Berger M, Wagner TH and Baker LC. Internet use and stigmatized illness. Soc Sci Med 2005; 61: 1821-1827.

105. Burns JM, Davenport TA, Durkin LA, et al. The Internet as a setting for mental health service utilisation by young people. Med J Aust 2010; 192: S22-S26.

106. Watson M, Jones D and Burns L. Internet research and informed consent: an ethical model for using archived emails. Int J Ther Rehabil 2007; 14: 396-403.

107. Shaw B and Post FR. A moral basis for corporate philanthropy. J Bus Ethics 1993; 12: 745-751.

108. Wootton BM, Titov N, Dear BF, et al. The acceptability of Internet-based treatment and characteristics of an adult sample with obsessive compulsive disorder: an Internet survey. PLoS ONE 2011; 6: e20548.

109. Kenwright M, Marks I, Gega L, et al. Computer-aided self-help for phobia/panic via Internet at home: a pilot study. Br J Psychiatry 2004; 184: 448-449.

110. Haemmerli $\mathrm{K}, \mathrm{Znoj} \mathrm{H}$ and Berger T. Internet-based support for infertile patients: a randomized controlled study. J Behav Med 2010; 33: 135-146.

111. Chana N, Kennedy P and Chessell ZJ. Nursing staffs' emotional well-being and caring behaviours. $J$ Clin Nurs 2015; 24: 2835-2848.

112. Mastracci S and Hsieh C. Emotional labor and job stress in caring professions: exploring universalism and particularism in construct and culture. Int J Public Admin. Epub ahead of print 4 March 2016. DOI: 10.1080/ 01900692.2015.1068327.

113. Dasan S, Gohil P, Cornelius V, et al. Prevalence, causes and consequences of compassion satisfaction and compassion fatigue in emergency care: a mixed-methods study of UK NHS consultants. Emerg Med J 2015; 32: $588-594$.

114. Mo PK and Coulson NS. Are online support groups always beneficial? A qualitative exploration of the empowering and disempowering processes of participation within HIV/AIDS-related online support groups. Int J Nurs Stud 2014; 51: 983-993.

115. International Confederation of Midwives (ICM). International code of ethics for midwives, 2014, http://www.in ternationalmidwives.org/assets/uploads/documents/CoreDocuments/CD2008_001\%20V2014\%20ENG \%20International\%20Code\%20of\%20Ethics\%20for\%20Midwives.pdf

116. Klitzman R and Bayer R. Mortal secrets: truth and lies in the age of AIDS. Baltimore, MD: Johns Hopkins University Press, 2003. 